\title{
Uniqueness of Meromorphic Functions Whose Differential Polynomials Share One Value
}

\author{
Jin Tao, Xinli Wang \\ College of Science, University of Shanghai for Science and Technology, Shanghai, China \\ Email:2609873599@qq.com
}

How to cite this paper: Tao, J. and Wang, X.L. (2018) Uniqueness of Meromorphic Functions Whose Differential Polynomials Share One Value. Journal of Applied Mathematics and Physics, 6, 2264-2272. https://doi.org/10.4236/jamp.2018.611188

Received: September 29, 2018

Accepted: November 16, 2018

Published: November 19, 2018

Copyright $\odot 2018$ by authors and Scientific Research Publishing Inc. This work is licensed under the Creative Commons Attribution International License (CC BY 4.0).

http://creativecommons.org/licenses/by/4.0/

\begin{abstract}
In this paper, we prove a uniqueness theorem of meromorphic functions whose some nonlinear differential shares 1 IM with powers of the meromorphic functions, where the degrees of the powers are equal to those of the nonlinear differential polynomials. This result improves the corresponding one given by Zhang and Yang, and other authors.
\end{abstract}

\section{Keywords}

Differential Polynomials, Meromorphic Functions, Uniqueness Theorems

\section{Introduction}

The meromorphic function mentioned in this paper refers to the meromorphic function over the entire complex plane. Let $f$ and $g$ be two non-constant meromorphic functions. $E \subset(0, \infty)$ means Linear measure finite set. $S(r, f)$ means $S(r, f)=o\{T(r, f)\},(r \rightarrow \infty, r \notin E)$. CM is the abbreviation of common multiplicities. And $I M$ is the abbreviation of ignored multiplicities. These concepts can be found in the literature [1]. Let $a$ be a finite complex number, if $f-a$ and $g-a$ have the same zero point and the same number of weights, then $f$ and $g C M$ share $a$. If $f-a$ and $g-a$ have the same zero point without counting the number, then $f$ and $g I M$ share $a$ [2]. In addition, the following definitions are required: let $p$ be a positive integer, and $a \in C \cup\{\infty\}$. Next $N_{p)}\left(r, \frac{1}{f-a}\right)$ means $f$ has a weight less than $p$ count function of the weight of the value point a within $|z|<r . \bar{N}_{p)}\left(r, \frac{1}{f-a}\right)$ means corresponding reduced count function; $N_{(p}\left(r, \frac{1}{f-a}\right)$ means the weight of $f$ is not less than $p$ count 
function of the weight of the value point a within $|z|<r . \bar{N}_{(p}\left(r, \frac{1}{f-a}\right)$ means corresponding reduced count function. Suppose $k$ is a non-negative number. Mark $N_{k}\left(r, \frac{1}{f-a}\right)$ defined as follows. See the literature [3] for details.

$$
N_{k}\left(r, \frac{1}{f-a}\right)=\bar{N}\left(r, \frac{1}{f-a}\right)+\bar{N}_{(2}\left(r, \frac{1}{f-a}\right)+\cdots+\bar{N}_{(k}\left(r, \frac{1}{f-a}\right)
$$

Before, Xiaomin Li and Zhitao Wen expanded Jilong Zhang's theorem, where $\left(f^{n}\right)^{\prime}$ changes to $f^{n-1} f^{(k)}$, so when $k=1$, that is Zhang's theorem. Similarly, in this paper, we continuously change $f^{n-1} f^{(k)}$ to $f^{n-1}(f-1) f^{(k)}$, which contained $f-1$. So we expended Xiaomin Li and Zhitao Wen's theorem.

In 2008, Lianzhong Yang and Jilong Zhang proved the following theorems:

Theorem A [4] Suppose $f$ is a non-constant entire function, $n \geq 7$ is a positive integer, if $f^{n}$ and $\left(f^{n}\right)^{\prime} C M$ share 1 , then $f^{n}=\left(f^{n}\right)^{\prime}$.

Theorem B [4] Suppose $f$ is a non-constant meromorphic function, $n \geq 12$ is a positive integer, if $f^{n}$ and $\left(f^{n}\right)^{\prime} C M$ share 1 , then $f^{n}=\left(f^{n}\right)^{\prime}$.

Recently Zhang Jilong improved the above theorem. Get the following result:

Theorem C [5] Suppose $f$ is a non-constant entire function, $n \geq 3$ is a positive integer, if $f^{n}$ and $\left(f^{n}\right)^{\prime} C M$ share 1 , then $f^{n}=\left(f^{n}\right)^{\prime}$.

Theorem $\mathrm{D}$ [5] Suppose $f$ is a non-constant meromorphic function, $n \geq 4$ is a positive integer, if $f^{n}$ and $\left(f^{n}\right)^{\prime} C M$ share 1 , then $f^{n}=\left(f^{n}\right)^{\prime}$.

Li Xiaomin and Wen Zhitao have improved on the basis of Zhang Jilong's theorem, as follows.

Theorem E [5] Suppose $f$ is a non-constant meromorphic function, $k$ is a positive integer, $n$ is a positive integer and satisfies $2 n \geq k+3+\sqrt{k^{2}+2 k+9}$, if $f^{n}$ and $\left(f^{n}\right)^{\prime} C M$ share 1 , then $f^{n}=\left(f^{n}\right)^{\prime}$.

Theorem $\mathbf{F}$ [5] Suppose $f$ is a non-constant meromorphic function, $k$ is a positive integer, $n$ is a positive integer and satisfies $2 n \geq 3 k+8+\sqrt{9 k^{2}+40 k+64}$, if $f^{n}$ and $\left(f^{n}\right)^{\prime}$ IM share 1 , then $f^{n}=\left(f^{n}\right)^{\prime}$.

Now we mainly improve the theorem of Li Xiaomin. Which that changes $f^{n}$ and $f^{n-1} f^{(k)}$ to $f^{n}(f-1)$ and $f^{n-1}(f-1) f^{(k)}$. We get the following theorem:

Theorem 1 Suppose $f$ is a non-constant meromorphic function, $k$ is a positive integer, $n$ is a positive integer and satisfies $2 n \geq 3 k+13+\sqrt{9 k^{2}+62 k+129}$, if $f^{n}(f-1)$ and $f^{n-1}(f-1) f^{(k)} I M$ share 1 , and the zeros of $f-1$ with multiplicity 2 at least., then $f=f^{(k)}$.

\section{Some Lemmas}

Lemma 1 [6] Suppose $F$ and $G$ are non-constant meromorphic functions, let

$$
H=\left(\frac{F^{\prime \prime}}{F^{\prime}}-2 \frac{F^{\prime}}{F-1}\right)-\left(\frac{G^{\prime \prime}}{G^{\prime}}-2 \frac{G^{\prime}}{G-1}\right)
$$

and suppose $H \neq 0$, if $F$ and $G I M$ share 1 , then 


$$
N_{E}^{1)}\left(r, \frac{1}{G-1}\right) \leq N(r, H)+S(r, F)+S(r, G)
$$

Lemma 2 [7] Let $f$ be a non-constant meromorphic function, and

$$
P(f)=a_{n} f^{n}+a_{n-1} f^{n-1}+\cdots+a_{1} f
$$

where $a_{1}, a_{2}, \cdots, a_{n-1}, a_{n}(\neq 0)$ are constants, then $T(r, P(f))=n T(r, f)+O(1)$.

Lemma 3 Let $f$ be a non-constant meromorphic function, $k(\geq 1)$ and $n(\geq 2)$ are two positive integers. Let $F=f^{n}(f-1)$ and $G=f^{n-1}(f-1) f^{(k)}$. If $F$ and $G I M$ share 1 , then $S(r, F)=S(r, G)=S(r, f)$.

Proof According to Lemma 2, we obtain

$$
T(r, F)=(n+1) T(r, f)+O(1)
$$

It can be seen from the above formula,

$$
S(r, F)=S(r, f)
$$

due to $G=f^{n-1}(f-1) f^{(k)}$, we have

$$
\begin{aligned}
T(r, G) & \leq(n-1) T(r, f)+T(r, f-1)+T\left(r, f^{(k)}\right) \\
& \leq(n-1) T(r, f)+T(r, f)+k \bar{N}(r, f)+S(r, f) \\
& \leq(n+k) T(r, f)+S(r, f)
\end{aligned}
$$

According to the second basic theorem and (2)

$$
\begin{aligned}
T(r, F) & \leq \bar{N}\left(r, \frac{1}{F-1}\right)+\bar{N}\left(r, \frac{1}{F}\right)+\bar{N}(r, F)+S(r, F) \\
& =\bar{N}\left(r, \frac{1}{G-1}\right)+\bar{N}\left(r, \frac{1}{f}\right)+\bar{N}(r, f)+S(r, f) \\
& \leq T(r, G)+2 T(r, f)+S(r, f)
\end{aligned}
$$

The above formula is combined with (1) to get

$$
T(r, G) \geq(n-1) T(r, f)+S(r, f)
$$

According to (3) and (4), we have

$$
S(r, G)=S(r, f)
$$

According to (2) and (5), we can get the conclusion of Lemma 3.

Lemma 4 [8] Let $f$ be a non-constant meromorphic function, $k, p$ are two positive integers. The zero point of $f-1$ is at least 2 , then

$$
\begin{gathered}
N_{p}\left(r, \frac{1}{f^{(k)}}\right) \leq k \bar{N}(r, f)+N_{p+k}\left(r, \frac{1}{f}\right)+S(r, f) \\
\bar{N}\left(r, \frac{1}{f-1}\right) \leq N\left(r, \frac{f}{f^{\prime}}\right) \leq \bar{N}(r, f)+\bar{N}\left(r, \frac{1}{f}\right)+S(r, f)
\end{gathered}
$$

Lemma 5 Let $f$ be a non-constant meromorphic function, $n(\geq 2), p$ are two positive integers. The zero point of $f-1$ is at least 2 . Let $F=f^{n}(f-1)$ and $G=f^{n-1}(f-1) f^{(k)}$, if $F$ and $G$ IM share 1 , then 
a) $\bar{N}\left(r, \frac{1}{G}\right) \leq(k+3) \bar{N}\left(r, \frac{1}{f}\right)+(k+1) \bar{N}(r, f)+S(r, f)$

b) $\bar{N}_{L}\left(r, \frac{1}{F-1}\right) \leq 2 \bar{N}\left(r, \frac{1}{f}\right)+2 \bar{N}(r, f)+S(r, f)$;

c) $\bar{N}_{L}\left(r, \frac{1}{G-1}\right) \leq(k+3) \bar{N}\left(r, \frac{1}{f}\right)+(k+2) \bar{N}(r, f)+S(r, f)$.

Proof According to Lemma 4, we have

$$
\begin{aligned}
\bar{N}\left(r, \frac{1}{G}\right) & \leq \bar{N}\left(r, \frac{1}{f}\right)+\bar{N}\left(r, \frac{1}{f-1}\right)+N_{1}\left(r, \frac{1}{f^{(k)}}\right)+S(r, f) \\
& \leq \bar{N}\left(r, \frac{1}{f}\right)+\bar{N}(r, f)+\bar{N}\left(r, \frac{1}{f}\right)+N_{k+1}\left(r, \frac{1}{f}\right)+k \bar{N}(r, f)+S(r, f) \\
& \leq(k+3) \bar{N}\left(r, \frac{1}{f}\right)+(k+1) \bar{N}(r, f)+S(r, f)
\end{aligned}
$$

This leads to the conclusion (a), obtained from the definition of the $N_{L}\left(r, \frac{1}{F-1}\right)$ and Lemma 3:

$$
\begin{aligned}
N_{L}\left(r, \frac{1}{F-1}\right) & \leq N\left(r, \frac{F}{F^{\prime}}\right) \leq T\left(r, \frac{F^{\prime}}{F}\right)+O(1)=m\left(r, \frac{F^{\prime}}{F}\right)+N\left(r, \frac{F^{\prime}}{F}\right)+O(1) \\
& =\bar{N}(r, F)+\bar{N}\left(r, \frac{1}{F}\right)+S(r, F)=2 \bar{N}(r, f)+2 \bar{N}\left(r, \frac{1}{f}\right)+S(r, f)
\end{aligned}
$$

This leads to conclusions (b), the same reason

$$
\bar{N}_{L}\left(r, \frac{1}{G-1}\right) \leq \bar{N}\left(r, \frac{1}{G}\right)+\bar{N}(r, G)+S(r, f)
$$

Combine $G=f^{n-1}(f-1) f^{(k)}$ and the q form in Lemma 5, we can get (c).

Lemma 6 Suppose $F$ and $G$ are non-constant meromorphic functions, and satisfy $\bar{N}(r, F)+\bar{N}\left(r, \frac{1}{F}\right)=S(r, F)$ and $\bar{N}(r, G)+\bar{N}\left(r, \frac{1}{G}\right)=S(r, G)$. If $F$ and $G I M$ share a non-zero constant $a$, then $F=G$ or $F G=1$.

\section{Proof}

$$
\text { Suppose } F=f^{n}(f-1), \quad G=f^{n-1}(f-1) f^{(k)} .
$$

Let $H$ be defined by Lemma 1 . The following two discussions,

Case 1 Suppose $H \neq 0$, then $F \neq G$, let

$$
V=\left(\frac{F^{\prime}}{F-1}-\frac{F^{\prime}}{F}\right)-\left(\frac{G^{\prime}}{G-1}-\frac{G^{\prime}}{G}\right)
$$

If $V=0$,

$$
1-\frac{1}{F}=B-\frac{B}{G}
$$

where $B \neq 0$ is a constant, if $\bar{N}(r, f) \neq S(r, f)$.

By (6) and (8), we get $B=1$.

So $F=G$, contradictory with the assumption of case 1 . 
Therefore, $\bar{N}(r, f)=S(r, f)$.

So $B \neq 1$, by (8), we get

$$
\begin{gathered}
G=\frac{B F}{(B-1) F+1}=\frac{B F}{F-\frac{1}{1-B}}\left(\frac{1}{B-1}\right) \\
\bar{N}\left(r, \frac{1}{F-\frac{1}{B-1}}\right)=S(r, f)
\end{gathered}
$$

According to the second basic theorem and (6) (8) (9) (10) we get

$$
\begin{aligned}
T(r, F) & \leq \bar{N}\left(r, \frac{1}{F}\right)+\bar{N}\left(r, \frac{1}{F-\frac{1}{B-1}}\right)+\bar{N}(r, F)+S(r, F) \\
& \leq \bar{N}\left(r, \frac{1}{f}\right)+\bar{N}\left(r, \frac{1}{f-1}\right)+S(r, f) \\
& \leq 2 \bar{N}(r, f)+S(r, f)
\end{aligned}
$$

By lemma 2, we have

$$
T(r, F)=(n+1) T(r, f)+O(1)
$$

Then

$$
(n+1) T(r, f)-3 T(r, f)=S(r, f)
$$

If $B=1$, then $F=G$, contradiction.

If $V$ was not always equal to $0,(7)$ can be rewritten into

$$
V=\frac{F^{\prime}}{F(F-1)}-\frac{G^{\prime}}{G(G-1)}
$$

Suppose $z_{0}$ is a pole of $f$ with multiplicity $p$, then $z_{0}$ is pole of $F$ with multiplicity $(n+1) p$. and $z_{0}$ is zero of $\frac{F^{\prime}}{F(F-1)}$ with multiplicity $(n+1) p-1$ at least. $z_{0}$ is zero of $\frac{G^{\prime}}{G(G-1)}$ with multiplicity $(n+1) p+k-1$ at least.

So $z_{0}$ is a zero of $V$ with multiplicity $\geq n$ at least.

$$
\begin{aligned}
n \bar{N}(r, f) \leq & N\left(r, \frac{1}{V}\right)+S(r, f) \leq N(r, V)+S(r, f) \\
\leq & \bar{N}\left(r, \frac{1}{G}\right)+\bar{N}_{L}\left(r, \frac{1}{F-1}\right)+\bar{N}_{L}\left(r, \frac{1}{G-1}\right)+S(r, f) \\
\leq & (k+3) \bar{N}\left(r, \frac{1}{f}\right)+(k+1) \bar{N}(r, f)+2 \bar{N}(r, f) \\
& +2 \bar{N}\left(r, \frac{1}{f}\right)+(k+3) \bar{N}\left(r, \frac{1}{f}\right)+(k+2) \bar{N}(r, f)+S(r, f) \\
\leq & (2 k+8) \bar{N}\left(r, \frac{1}{f}\right)+(2 k+5) \bar{N}(r, f)+S(r, f)
\end{aligned}
$$


Then

$$
(n-2 k-5) \bar{N}(r, f) \leq(2 k+8) \bar{N}\left(r, \frac{1}{f}\right)+S(r, f)
$$

The following two sub-cases are discussed:

Sub-case 1.1 suppose

$$
U=\frac{F^{\prime}}{F-1}-\frac{G^{\prime}}{G-1}
$$

If $U=0$, we have

$$
F=D G+1-D
$$

where $D \neq 0$ is a constant. Then

$$
\bar{N}(r, f)=S(r, f) .
$$

Suppose $\bar{N}\left(r, \frac{1}{f}\right) \neq S(r, f), \quad D=1, \quad F=G$, contradiction;

Suppose $\bar{N}\left(r, \frac{1}{f-1}\right) \neq S(r, f), \quad D=1, \quad F=G$, contradiction.

$$
\text { So } \bar{N}\left(r, \frac{1}{f}\right)=S(r, f), \bar{N}\left(r, \frac{1}{f-1}\right)=S(r, f) \text {. }
$$

If $D \neq 1, \bar{N}\left(r, \frac{1}{G}\right)=\bar{N}\left(r, \frac{1}{F+D-1}\right)$,

$$
\bar{N}\left(r, \frac{1}{G}\right)=\bar{N}\left(r, \frac{1}{f}\right)+\bar{N}\left(r, \frac{1}{f^{(k)}}\right)+\bar{N}\left(r, \frac{1}{f-1}\right)=S(r, f)
$$

So $\bar{N}\left(r, \frac{1}{F+D-1}\right)=S(r, f)$.

Then

$$
\begin{aligned}
(n+1) T(r, f) & =T(r, F)+S(r, f) \\
& \leq \bar{N}\left(r, \frac{1}{F}\right)+\bar{N}\left(r, \frac{1}{F+D-1}\right)+\bar{N}(r, F)+S(r, f) \\
& =S(r, f)
\end{aligned}
$$

Obviously impossible.

Suppose $U$ is not always equal to 0 , let $z_{1}$ be a zero of $f$ with multiplicity $q$, then $z_{1}$ is a zero $F$ with multiplicity $n q$ and $z_{1}$ is zero of $\frac{F^{\prime}}{F-1}$ with multiplicity $n q-1$ at least. $z_{0}$ is zero of $\frac{G^{\prime}}{G-1}$ with multiplicity $(n-1) q-1$ at least.

So $z_{1}$ is a zero of $U$ with multiplicity $n-2$ at least.

$$
\begin{aligned}
(n-2) \bar{N}\left(r, \frac{1}{f}\right) & \leq N\left(r, \frac{1}{U}\right)+S(r, f) \leq N(r, U)+S(r, f) \\
& \leq \bar{N}(r, f)+\bar{N}_{L}\left(r, \frac{1}{F-1}\right)+\bar{N}_{L}\left(r, \frac{1}{G-1}\right)+S(r, f) \\
& \leq(k+5) \bar{N}\left(r, \frac{1}{f}\right)+(k+4) \bar{N}(r, f)+S(r, f)
\end{aligned}
$$


So

$$
(n-k-7) \bar{N}\left(r, \frac{1}{f}\right) \leq(k+5) \bar{N}(r, f)+S(r, f)
$$

Also

$$
\begin{aligned}
T(r, F) & \leq \bar{N}\left(r, \frac{1}{F}\right)+\bar{N}\left(r, \frac{1}{F-1}\right)+\bar{N}(r, F)+S(r, F) \\
& \leq 2 \bar{N}\left(r, \frac{1}{f}\right)+2 \bar{N}(r, f)+\bar{N}\left(r, \frac{1}{F-1}\right)+S(r, f) \\
\bar{N}\left(r, \frac{1}{F-1}\right) & \leq \bar{N}\left(r, \frac{f^{n-1}(f-1) f^{(k)}}{f^{n}(f-1)}\right) \leq T\left(r, \frac{f^{(k)}}{f}\right)+S(r, f) \\
& =m\left(r, \frac{f^{(k)}}{f}\right)+\bar{N}\left(r, \frac{f^{(k)}}{f}\right)+S(r, f) \\
& \leq k \bar{N}(r, f)+k \bar{N}\left(r, \frac{1}{f}\right)+S(r, f)
\end{aligned}
$$

Then

$$
T(r, F) \leq(k+2) \bar{N}\left(r, \frac{1}{f}\right)+(k+2) \bar{N}(r, f)+S(r, f)
$$

If $n-2 k-5>0$, and $\bar{N}\left(r, \frac{1}{f}\right)=S(r, f), \bar{N}(r, f)=S(r, f)$ one of the two forms is established. Then $\bar{N}\left(r, \frac{1}{f}\right)+\bar{N}(r, f)=S(r, f)$, substituting the above formula is obviously impossible.

Or $T(r, F)=S(r, f)$, contradiction.

So $\bar{N}\left(r, \frac{1}{f}\right)+\bar{N}(r, f) \neq S(r, f)$, we get

$$
\begin{aligned}
\bar{N}\left(r, \frac{1}{f}\right) \neq S(r, f), \bar{N}(r, f) \neq S(r, f), \text { then } \\
(n-k-7) \bar{N}\left(r, \frac{1}{f}\right) \leq \frac{(k+4)(2 k+8)}{n-2 k-6} \bar{N}\left(r, \frac{1}{f}\right)+S(r, f) \\
(n-k-7) \leq \frac{(k+4)(2 k+8)}{n-2 k-6} \\
\frac{3 k+13-\sqrt{9 k^{2}+62 k+129}}{2} \leq n \leq \frac{3 k+13+\sqrt{9 k^{2}+62 k+129}}{2}
\end{aligned}
$$

Case 2

Situation 2.1 
If $\bar{N}\left(r, \frac{1}{f}\right)+\bar{N}(r, f)=S(r, f)$, we get

$$
\bar{N}\left(r, \frac{1}{F}\right)+\bar{N}(r, F)=S(r, F), \quad \bar{N}\left(r, \frac{1}{G}\right)+\bar{N}(r, G)=S(r, F)
$$

According to Lemma 6, we get $F=G$ or $F G=1$.

Firstly, if $F=G, f^{n}(f-1)=f^{n-1}(f-1) f^{(k)}, f=f^{(k)}$, conclusion established.

Secondly, if $F G=1, f^{n}(f-1) \cdot f^{n-1}(f-1) f^{(k)}=1, f^{2 n-1}(f-1)^{2} f^{(k)}=1$.

Obviously $f$ is entire function.

And

$$
\begin{aligned}
& T\left(r, f^{2 n-1}\right) \leq \bar{N}\left(r, \frac{1}{f^{(k)}}\right)+\bar{N}\left(r, \frac{1}{(f-1)^{2}}\right)+S(r, f) \\
&=T(r, f)+2 T(r, f)+S(r, f) \\
&=3 T(r, f)+S(r, f) \\
&(2 n-1) T(r, f) \leq 3 T(r, f)+S(r, f)
\end{aligned}
$$

$T(r, f)=S(r, f)$, contradiction.

Situation 2.2

If $\bar{N}(r, f) \neq S(r, f)$, we get $B=0$.

So $G-1=A(F-1)$.

If $\bar{N}(r, f) \neq S(r, f)$, we get $A=1$, then $F=G$.

And if $\bar{N}(r, f)=S(r, f)$,

$$
\begin{aligned}
& T(r, F) \leq \bar{N}\left(r, \frac{1}{f}\right)+\bar{N}(r, f)+\bar{N}\left(r, \frac{1}{f-1}\right)+\bar{N}\left(r, \frac{1}{F-1}\right)+S(r, f) \\
& \leq(k+2) \bar{N}(r, f)+S(r, f) \\
&(n+1) T(r, f) \leq(k+2) \bar{N}(r, f)+S(r, f)
\end{aligned}
$$

We get $n \leq k+1$, that contradict with $2 n \geq 3 k+13+\sqrt{9 k^{2}+62 k+129}$.

Suppose $\bar{N}(r, f)=S(r, f)$,

If $\bar{N}\left(r, \frac{1}{f}\right) \neq S(r, f)$ we get $B=A-1$.

If $A=1$, we get $B=0$, then $F=G$.

If $A \neq 1$, we get

$$
\begin{gathered}
F-1=\frac{G-1}{G-\frac{1}{A-1}}\left(\frac{1}{A-1}\right) \\
\bar{N}(r, f)=\bar{N}(r, G)=\bar{N}\left(r, \frac{1}{G-\frac{1}{A-1}}\right)=S(r, f)
\end{gathered}
$$

And $T(r, F)=T(r, G)+O(1)$ 


$$
\begin{aligned}
(n+1) T(r, f) & =T(r, F)+O(1)=T(r, G) \\
& \leq \bar{N}(r, G)+\bar{N}\left(r, \frac{1}{G}\right)+\bar{N}\left(r, \frac{1}{\left.G-\frac{1}{A-1}\right)}\right) S(r, f) \\
& \leq \bar{N}\left(r, \frac{1}{G}\right)+S(r, f) \leq(k+3) \bar{N}\left(r, \frac{1}{f}\right)+S(r, f)
\end{aligned}
$$

We get $n \leq k+2$, which contradicts with $2 n \geq 3 k+13+\sqrt{9 k^{2}+62 k+129}$. Therefore, Theorem 1 is proved.

\section{Conflicts of Interest}

The authors declare no conflicts of interest regarding the publication of this paper.

\section{References}

[1] Hayman, W.K. (1964) Meromorphic Functions. Claredon Press, Oxford.

[2] Yi, H.X. and Yang, C.J. (1995) Theorem on the Uniqueness of Meromorphic Functions. Science Press, Beijing.

[3] Alzahary, T.C. and Yi, H.X. (2004) Weighted Sharing Three Values and Uniqueness of Meromorphic Functions. Journal of Mathematical Analysis and Applications, 295, 247-257. https://doi.org/10.1016/j.jmaa.2004.03.040

[4] Yang, L.Z. and Zhang, J.L. (2008) Non-Existence of Meromorphic Solusions of Fermat Type Functional Equation. Aequationes Mathematicae, 76, 140-150. https://doi.org/10.1007/s00010-007-2913-7

[5] Yang, L.Z. and Zhang, J.L. (2009) A Power of a Meromorphic Function Sharing a Small Function Sharing a Small Function with Its Derivative. Annales Academiae Scientiarum Fennicae Mathematica, 34, 249-260.

[6] Yi, H.X. (1997) Uniqueness Theorems for Meromorphic Functions Whose n-th Derivatives Share the Same 1-Points. Complex Variables, Theory and Application, 34, 421-436. https://doi.org/10.1080/17476939708815064

[7] Yang, C.C. (1972) On Deficiencies of Differential Polynomial. Mathematische Zeitschrift, 125, 107-112. https://doi.org/10.1007/BF01110921

[8] Lahiri, J. and Sarkar, A. (2004) Uniqueness of a Meromorphic Function and Its Derivative. Journal of Inequalities in Pure and Applied Mathematics, 5, 20-21. 